\title{
THE FUNDAMENTAL GROUP OF ONE-DIMENSIONAL SPACES
}

\author{
M. L. CURTIS ${ }^{1}$ AND M. K. FORT, JR. ${ }^{1}$
}

1. Introduction.Throughout, $X$ is a one-dimensional separable metric space. It is shown in [1] that $\pi_{n}(X)=0$ for all $n>1$, and it follows that $\pi_{1}(X)$ determines the singular homology groups of $X$ (see [2, p. 481]). Examples show that $\pi_{1}(X)$ need not be free (see $[4$, p. 455$])$, but it may well be that the finitely-generated subgroups of $\pi_{1}(X)$ are free. ${ }^{2}$ If this is the case, it follows that all of the singular homology groups of $X$ vanish in dimensions higher than one. We study $\pi_{1}(X)$, and are able to obtain some information about it. The implications for the homology of $X$ are not yet known.

We first show that $\pi_{1}(X)$ has no elements of finite order. Next, let $S$ be the space which is the union of the circles

$$
S_{n}=\left\{(x, y) \mid\left(x-\frac{1}{n}\right)^{2}+y^{2}=\frac{1}{n^{2}}\right\}
$$

in the plane. We show in $\$ 2$ that if $X$ is also a locally connected continuum, then either $\pi_{1}(X)$ is finitely-generated (and free) or $\pi_{1}(X)$ contains a subgroup isomorphic with $\pi_{1}(S)$. This follows by showing: (1) If $Y$ is a subset of $X$, then $\pi_{1}(Y)$ is a subgroup of $\pi_{1}(X)$, and (2) $X$ is either locally simply connected or it contains a subset having the homotopy type of $S$.

$\S \S 3$ and 4 are devoted to showing that $\pi_{1}(X)$ is cyclically-abelian; i.e. its only finitely-generated abelian subgroups are cyclic. In $\S 3$, it is shown that each element of $\pi_{1}(X)$ contains a special kind of representative which we call normal, and that two such representatives represent the same element of $\pi_{1}(X)$ if and only if they are Fréchet equivalent. In $\$ 4$ we show that two normal loops commute (up to homotopy) if and only if they are both multiples of one loop.

2. Basic properties of $\pi_{1}(X)$.

LEMma 2.1. $\pi_{1}(X)$ has no elements of finite order.

Presented to the Society, November 30, 1957; received by the editors April 21, 1958.

${ }^{1}$ Supported in part by National Science Foundation Grants No. G4211 and No. G3016.

${ }^{2}$ Added in proof. The authors have since proved that this is the case using methods considerably different from those employed in the present paper. 
Proof. Suppose that there exists a map $f:\left(S^{1}, y_{0}\right) \rightarrow\left(X, x_{0}\right)$ such that $[f] \in \pi_{1}\left(X, x_{0}\right)$ has order $n$. Let $g:\left(S^{1}, y_{0}\right) \rightarrow\left(X, x_{0}\right)$ be the map obtained by dividing the boundary $S^{1}$ of the disk $D$ into $n$ equal parts and applying $f$ to each part. We can extend $g$ to a map $G: D \rightarrow X$.

Let $K$ be the space obtained from $D$ by identifying the corresponding points of the $n$ parts of the boundary of $D$. Then $G$ can be factored as $G=B A, D \rightarrow^{A} K \rightarrow^{B} X$, where $A$ is the identification map. A routine calculation gives $\pi_{1}(K)=Z_{n}$ (integers modulo $n$ ) so that $H_{1}(K, Z)$ $=Z_{n}$. It follows from the theorem in [1] that $B$ is homotopic to a constant. Hence $f$ is homotopic to a constant, and the lemma is proved.

The next lemma gives an important property of one-dimensional spaces which is used repeatedly in the remainder of this paper.

Lemma 2.2. If $f: S^{1} \rightarrow X$ is homotopic to a constant, then there is a $F: S^{1} \times I \rightarrow X$ such that $F_{0}=f, F_{1}\left(S^{1}\right)$ is a single point, and $F\left(S^{1} \times I\right)$ $=f\left(S^{1}\right)$.

Proof. $f$ can be factored as $f=g h, S^{1} \rightarrow^{h} D \rightarrow^{0} X$, where $D$ is a dendrite (see [3]). Since $h\left(S^{1}\right)$ is a connected subset of a dendrite, it is contractible in itself to a point. The lemma follows.

Corollary 2.1. If $Y$ is a subspace of $X$, then $\pi_{1}(Y)$ is a subgroup of $\pi_{1}(X)$.

Of course, not all subgroups of $\pi_{1}(X)$ are realizable as fundamental groups of subspaces of $X$. For example, if $X$ consists of two circles with a single common point, then $\pi_{1}(X)$ is a free group with two generators, and it contains a subgroup with infinitely-many generators. This subgroup cannot be $\pi_{1}(Y)$ for any $Y \subset X$.

THEOREM 2.1. If a locally connected continuum $W$ is not locally simply connected, then it contains a subspace which has the homotopy type of $S$ (see the definition of $S$ in the introduction).

Proof. Let $x$ be a point at which $W$ is not locally simply connected.

CASE I. There exists a sequence $C_{1}, C_{2}, \cdots$ of disjoint simple closed curves converging to $x$ such that no $C_{i}$ contains $x$.

CASE Ia. Some arc $\alpha$ with end point $x$ intersects an infinite number of the $C_{i}$.

We linearly order $\alpha$ so that $x$ is the least point of $\alpha$. Let $y_{0}$ be the other end point of $\alpha$. We define $B_{0}$ to be the first member of the sequence $C_{1}, C_{2}, \ldots$ which intersects $\alpha$, and we define $x_{0}$ to be the least point of $\alpha$ in $B_{0}$. We define $B_{1}$ to be the first set $C_{i}$ in the sequence 
$C_{1}, C_{2}, \ldots$ such that $C_{i} \cap \alpha \neq \phi$ and $C_{i} \cap \alpha$ is contained in the subarc of $\alpha$ from $x$ to $x_{0}$. Let $x_{1}$ be the least point of $B_{1} \cap \alpha$ and let $y_{1}$ be the greatest point of $B_{1} \cap \alpha$. We continue this process, obtaining $B_{0}, B_{1}, B_{2}, \cdots$ and points $y_{0}, x_{0}, y_{1}, x_{1}, y_{2}, x_{2}, \ldots$. We let

$$
T=\left(\alpha \cup \bigcup_{i=0}^{\infty} B_{i}\right)-\bigcup_{i=0}^{\infty} \alpha_{i}
$$

where $\alpha_{i}$ is the open $\operatorname{arc}$ on $\alpha$ from $x_{i}$ to $y_{i}\left(\alpha_{i}=\phi\right.$ if $\left.x_{i}=y_{i}\right)$. The set $T$ obviously has the homotopy type of $S$.

CASE Ib. No arc with end point $x$ intersects an infinite number of the $C_{i}$.

Let $B_{1}=C_{1}$ and let $\alpha_{1}$ be an arc from $B_{1}$ to $x$ such that $\alpha_{1} \cap B_{1}$ consists of a single point. We choose a neighborhood $N_{1}^{\prime}$ of $x$ which has diameter less than 1 and does not intersect $B_{1}$. Then we choose a neighborhood $N_{1}$ of $x$ such that any two points of $N_{1}$ are joinable by an arc in $N_{1}^{\prime}$. We choose $B_{2}$ to be one of $\left\{C_{n}\right\}$ such that $B_{2} \subset N_{1}$ and $B_{3} \cap \alpha_{1}=\phi$. Then we let $\alpha_{2}$ be an arc in $N_{1}^{\prime}$ such that the end points of $\alpha_{2}$ are $\alpha_{2} \cap B_{2}$ and $\alpha_{2} \cap \alpha_{1}$. We next choose a neighborhood $N_{2}^{\prime}$ of $x$ which has diameter less than $1 / 2$ and does not intersect $B_{1} \cup B_{2}$. Then we choose a neighborhood $N_{2}$ of $x$ such that any two points of $N_{2}$ are joinable by an arc in $N_{2}^{\prime}$. We choose $B_{3}$ to be one of $\left\{C_{n}\right\}$ such that $B_{3} \subset N_{2}, B_{3} \cap\left(\alpha_{1} \cup \alpha_{2}\right)=\phi$ and $B_{3} \cap\left(B_{1} \cup B_{2}\right)=\phi$. Then we let $\alpha_{3}$ be an arc in $N_{2}^{\prime}$ such that the end points of $\alpha_{3}$ are $\alpha_{3} \cap B_{3}$ and $\alpha_{3} \cap\left(\alpha_{1} \cup \alpha_{2}\right)$. Continuing in this manner, we obtain a set

$$
V=\bigcup_{k=1}^{\infty}\left(B_{k} \cup \alpha_{k}\right)=D \cup \bigcup_{k=1}^{\infty} B_{k},
$$

where $D$ is a dendrite. It is easy to see that $V$ has the homotopy type of $S$.

CASE II. There does not exist a sequence of simple closed curves as in Case I.

In this case we observe that there exists a sequence $C_{i}$ of simple closed curves such that $\left\{\right.$ diameter $\left.C_{i}\right\} \rightarrow 0$ and each $C_{i}$ contains $x$.

CASE IIa. There exists an arc $\alpha$ with end point $x$ such that $\alpha$ intersects infinitely many of the $C_{i}$ at points other than $x$.

In this case, there exists a subsequence $\left\{C_{i}^{\prime}\right\}$ of $\left\{C_{i}\right\}$ such that $\left(\alpha \cap C_{i}^{\prime}\right)-x$ is nonempty and connected, for otherwise we would be in Case I. Each $C_{i}^{\prime}$ contains an arc $A_{i}$ such that $A_{i} \cap \alpha$ consists of precisely the end points of $A_{i}$. We can choose a subsequence $\left\{A_{i}^{\prime}\right\}$ of $\left\{A_{i}\right\}$ such that $A_{j}^{\prime} \cap A_{k}^{\prime}=x$ for $j \neq k$, for otherwise we would be in Case I. Then $\alpha \cup \bigcup_{i=1}^{\infty} A_{i}^{\prime}$ has the homotopy type of $S$.

CASE IIb. No arc with end point $x$ intersects an infinite number of the $C_{i}$. 
In this case, the type of arguments used above yield a subspace of $W$ which is homeomorphic with $S$, so the theorem is established.

THEOREM 2.2. If $X$ is (also) a locally connected continuum, then $\pi_{1}(X)$ is free if and only if $X$ is locally simply connected.

Proof. If $X$ is locally simply connected, it is dominated by a finite one-dimensional polyhedron, and hence $\pi_{1}(X)$ is free. If $X$ is not locally simply connected, it follows from Theorem 2.1 that $X$ contains a subspace $Y$ with the homotopy type of $S$. By Corollary 2.1, $\pi_{1}(Y)$ is a subgroup of $\pi_{1}(X)$. Since $\pi_{1}(S)$ is not free (see [4, p. 455]) it follows that $\pi_{1}(X)$ is not free.

Corollary 2.2. If $X$ is (also) a locally connected continuum, then $\pi_{1}(X)$ is either finitely generated or uncountably generated.

\section{Normal loops and Fréchet equivalence.}

Definition. A loop $f: I \rightarrow W$ is normal if for each subinterval $J=[u, v]$ of $I$ for which $f(u)=f(v)$, if $f \mid J$ is inessential relative to $\{u, v\}$, then $f \mid J$ is constant.

\section{Lemma 3.1. Each element of $\pi_{1}(X)$ contains a normal loop.}

Proof. Let $f$ be a loop in $X$ with end points at $x_{0}$. Let $S$ be the collection of all open subsets $G$ of $I$ such that: If $(u, v)$ is a component of $G$, then $f(u)=f(v)$ and $f \mid[u, v]$ is homotopic to a constant relative to $\{u, v\} . S$ is partially ordered by inclusion, and we assert that $S$ contains a maximal element.

Let $C$ be any chain of $S$ and let $G$ be the union of the members of $C$. We shall show that $G$ is in $S$, and then application of Zorn's lemma will assure the existence of a maximal element of $S$.

It is easily seen that $C$ contains a cofinal sequence $G_{1} \subset G_{2}$ $\subset G_{3} \subset \ldots$. For each component $J$ of $G$, we can define a sequence $J_{1} \subset J_{2} \subset J_{3} \subset \cdots$ such that $J=\cup_{n=1}^{\infty} J_{n}$, and each $J_{n}$ is either empty or a component of $G_{n}$. It is convenient to select $J_{1}$ to be empty.

By Lemma 2.2 any loop which is homotopic to a constant map is homotopic in its range to a constant map. Hence for a component $J$ of $G$, there exists a sequence $f_{1}, f_{2}, f_{3}, \cdots$ of maps of $J$ into $X$ such that

(1) $f_{1}=f \mid \bar{J}$,

(2) $f_{n}\left|\left(\bar{J}-J_{n}\right)=f\right|\left(\bar{J}-J_{n}\right)$,

(3) $f_{n}$ is constant on $J_{n}$,

(4) $f_{n}$ can be joined to $f_{n+1}$ by a homotopy, the range of which is $f\left(\bar{J}-J_{n}\right)$.

The sequence $\left\{f_{i}\right\}$ converges to a constant map on $J$, and this constant map is homotopic to $f \mid \bar{J}$. Hence $G$ is in $S$. 
Let $G^{*}$ be a maximal element of $S$, and define $g$ to be the map which agrees with $f$ on $I-G^{*}$ and is constant on each component of $G^{*}$. Clearly $g$ is homotopic to $f$ and $g$ is normal.

Lemma 3.2. If $f: I \rightarrow W$ is a normal loop and $f$ can be factored as $f=g h, I \rightarrow^{h} D \rightarrow^{\circ} W$ where $D$ is a dendrite and $g$ is light, then $h$ is monotone.

Proof. Suppose $h\left(t_{1}\right)=h\left(t_{2}\right)$, and let $J=\left[t_{1}, t_{2}\right]$. Then $h \mid J$ is homotopic to a constant map relative to $\left\{t_{1}, t_{2}\right\}$, and since $f$ is normal, $f \mid J$ is a constant map. Since $g$ is light, $h \mid J$ must be constant so that $h$ is monotone.

Definition. Two maps $f_{1}, f_{2}: I \rightarrow W$ are Fréchet equivalent (see appendix) if there exist order-preserving monotone maps $m_{1}, m_{2}$ of $I$ onto $I$ such that $f_{1} m_{1}=f_{2} m_{2}$.

Theorem 3.1. Two normal loops from $x_{0}$ in $X$ represent the same element of $\pi_{1}\left(X, x_{0}\right)$ if and only if they are Fréchet equivalent.

Proof. If $f_{1}$ and $f_{2}$ are Fréchet equivalent normal loops, then they are clearly homotopic since $m_{1}$ and $m_{2}$ are homotopic to the identity on $I$.

Suppose $f_{1}$ and $f_{2}$ represent the same element of $\pi_{1}\left(X, x_{0}\right)$. Let $C$ denote the unit circle in the plane, $U$ its upper hemisphere and $L$ its lower hemisphere. Take homeomorphisms $h_{1}, h_{2}$ of $U, L$ respectively onto $I$ such that $h_{1}$ and $h_{2}$ agree on the two points of $U \cap L$. Define $F: C \rightarrow X$ by $F \mid U=f_{1} h_{1}$ and $F \mid L=f_{2} h_{2}$.

It follows from [3] that $F$ can be factored as $F=G H, C \rightarrow{ }^{B} D \rightarrow{ }^{a} X$, where $D$ is a dendrite and $G$ is light. Then $f_{1}=G H h_{1}^{-1}$ and $f_{2}=G H h_{2}^{-1}$. It follows from Lemma 3.2 that $H h_{1}^{-1}$ and $H h_{2}^{-1}$ are monotone maps into $D$. These maps agree on the endpoints of $I$ and must map $I$ onto the same arc in $D$ since the arc connecting a pair of points in a dendrite is unique. Then there exist order-preserving monotone maps $m_{1}, m_{2}$ of $I$ onto $I$ such that $H h_{1}^{-1} m_{1}=H h_{2}^{-1} m_{2}$ (see Appendix). Hence $f_{1} m_{1}=f_{2} m_{2}$, and the theorem is proved.

4. Commuting elements of $\pi_{1}(X)$. In this section it is convenient to consider a path to be a map of any nondegenerate closed interval into $X$. The statement that $f:[a, b] \rightarrow X$ and $g:[c, d] \rightarrow X$ are Fréchet equivalent means that there exist monotone orientation-preserving maps $m_{1}: I \rightarrow[a, b]$ and $m_{2}: I \rightarrow[c, d]$ such that $f m_{1}=g m_{2}$. We choose a metric in $X$ and use it throughout this section.

Definition. If $f: J \rightarrow X$ is a path, $A$ is a closed subset of $X$, and $\epsilon>0$, then the number $N(f, \epsilon, A)$ of $\epsilon$-excursions from $A$ is the number of components $C$ of $J-f^{-1}(A)$ for which the diameter of $f(C)$ is greater than $\epsilon$. 
This number has the following properties:

(1) $N(f, \epsilon, A)$ is always a non-negative integer.

(2) If $c \in[a, b]$, then $N(f \mid[a, c], \epsilon, A)+N(f \mid[c, b], \epsilon, A) \geqq N(f, \epsilon, A)$, and the equality holds if $c \in f^{-1}(A)$.

(3) If $f_{1}$ and $f_{2}$ are Fréchet equivalent, then $N\left(f_{1}, \epsilon, A\right)=N\left(f_{2}, \epsilon, A\right)$ for all $\epsilon>0$ and for all $A$.

Lemma 4.1. A light path $f:[a, b] \rightarrow X$ cannot be Fréchet equivalent to a restriction of itself to a proper subinterval.

Proof. Suppose $f$ is Fréchet equivalent to $f \mid[c, d]$. We define $A=\{f(c), f(d)\}$. Since $f$ is light (and we may assume without loss of generality that $d<b)$, there exists $\epsilon>0$ such that $N(f \mid[d, b], \epsilon, A)>0$. However, property (2) above implies that $N(f \mid[a, c], \epsilon, A)$ $+N(f \mid[c, d], \epsilon, A)+N(f \mid[d, b], \epsilon, A)=N(f, \epsilon, A)$, and property (3) implies that $N(f \mid[c, d], \epsilon, A)=N(f, \epsilon, A)$. This yields a contradiction.

Definition. By an $(\epsilon, A)$ set for a path $f: J \rightarrow X$ we mean a component $C$ of $J-f^{-1}(A)$ such that the diameter of $f(C)$ is greater than $\epsilon$.

The next lemma follows directly from the definitions.

Lemma 4.2. Suppose $f:[a, b] \rightarrow X$ and $g:[c, d] \rightarrow X$ are Fréchet equivalent and suppose $\epsilon>0$. If $t_{1}$ is the greatest number in $[a, b]$ that precedes each $(\epsilon, A)$ set for $f$, and $t_{2}$ is the greatest number in $[c, d]$ that precedes each $(\epsilon, A)$ set for $g$, then $f \mid\left[a, t_{1}\right]$ is Fréchet equivalent to $g \mid\left[c, t_{2}\right]$.

Definition. We say that paths $f:[a, b] \rightarrow X$ and $g:[c, d] \rightarrow X$ are coincident on an initial segment if there exist $b^{\prime} \in(a, b]$ and $d^{\prime} \in(c, d]$ such that $f \mid\left[a, b^{\prime}\right]$ is Fréchet equivalent to $g \mid\left[c, d^{\prime}\right]$.

Theorem 4.1. Let $f$ and $g$ be normal loops at $x_{0}$ in $X$. If the loops $f g$ and $g f$ represent the same element of $\pi_{1}\left(X, x_{0}\right)$, then $f$ and $g$ are both equivalent to powers of some loop $h$.

Proof. We let $f^{*}$ denote the inverse of the $\operatorname{loop} f$; i.e. if $f$ is defined on $[a, b]$ and $\theta:[a, b] \rightarrow[a, b]$ is the orientation-reversing isometry, then $f^{*}(t)=f(\theta(t))$ for all $t \in[a, b]$.

If $f, g, f^{*}$ have a coincident initial segment, we let $\lambda$ be a "maximal" path along which they coincide. By restricting $f$ and $g$ to appropriate subintervals of their domains, we obtain normal loops $\phi$ and $\psi$ at some point $p$ of $x$ such that $f \sim \lambda \phi \lambda^{*}$ and $g \sim \lambda \psi \lambda^{*}$. Since $\lambda$ was taken to be maximal, at least one of the loops $\phi \psi, \phi \psi^{*}, \phi^{*} \psi, \phi^{*} \psi^{*}$ must be normal; say $\phi \psi$ is normal. Since $f g \sim g f$, we have $\phi \psi \sim \psi \phi$.

We next define a mapping $F$ of $S^{1}$ into $X$ which is like $\phi \psi$ on the upper arc $s^{+}$of $S^{1}$ and like $\psi \phi$ on the lower arc $s^{-}$of $S^{1}$. We may factor 
$F=L M$ where $M$ maps $S^{1}$ into a dendrite and $L$ is light. $F \mid s^{+}$is normal and hence by Lemma 3.2, $M \mid s^{+}$is monotone. It follows that $\alpha=M\left(s^{+}\right)$is an arc. Since $M$ maps each of the right and left halves of $s^{-}$onto arcs in the dendrite, either $M\left(s^{-}\right)=\alpha$, or $M\left(s^{-}\right)=\alpha \cup \beta$ where $\beta$ is an arc whose intersection with $\alpha$ is an end point $q$ of $\beta$. We will show that this latter case cannot occur.

Suppose that a nondegenerate $\beta$ exists as in the previous paragraph. Then $q$ divides $\alpha$ into subarcs $\alpha_{1}$ and $\alpha_{2}$ (perhaps one of these will be degenerate). Choose $\epsilon>0$ so that $\epsilon$ is less than the diameter of $L(\beta)$, and let $A$ be the set consisting of the points $p$ and $L(q)$. We observe that ${ }^{3}$

$$
\begin{aligned}
& N\left(L \mid \alpha_{1}, \epsilon, A\right)+N\left(L \mid \alpha_{2}, \epsilon, A\right)=N\left(F \mid s^{+}, \epsilon, A\right) \\
& =N(\phi, \epsilon, A)+N(\psi, \epsilon, A)=N\left(F \mid s^{-}, \epsilon, A\right) \\
& =N\left(L \mid \alpha_{1} \cup \beta, \epsilon, A\right)+N\left(L \mid \alpha_{2} \cup \beta, \epsilon, A\right) \\
& =N\left(L \mid \alpha_{1}, \epsilon, A\right)+N\left(L \mid \alpha_{2}, \epsilon, A\right)+2 N(L \mid \beta, \epsilon, A) .
\end{aligned}
$$

Since $N(L \mid \beta, \epsilon, A) \neq 0$, this is impossible, and we now see that $M$ maps $s^{-}$onto the arc $\alpha$, and $M$ is monotone on each half of $s^{-}$.

Let $a$ and $d$ be the end points of $\alpha$ (which we will now think of as an interval on the real line). There are points $b$ and $c$ on $\alpha$ such that:

$$
\begin{array}{llll}
\phi \sim L \mid[a, b] & \left(=\phi_{1}\right), & & \psi L L \mid[b, d]\left(=\psi_{1}\right), \\
\psi \sim L \mid[a, c] & \left(=\psi_{2}\right), & & \phi \sim L \mid[c, d]\left(=\phi_{2}\right) .
\end{array}
$$

Furthermore, each of these restrictions of $L$ is normal.

We now choose $\epsilon>0$ small enough so that there exist $(\epsilon, p)$ sets for $L \mid \alpha$. We will prove by induction on the number $n$ of such sets that $\phi$ and $\psi$ are equivalent to powers of some loop.

Assume $n=1$. The single $(\epsilon, p)$ set $C$ must be contained in the domain of either $\phi_{1}$ or $\psi_{1}$; say it is in that of $\phi_{1}$. The domain of $\phi_{2}$ must also contain an $(\epsilon, p)$ set, and since we are assuming $n=1$, it contains $C$. (See the figure on the following page.) This implies that $c<b$. We let $k$ be the left limit point of $C$. Then by Lemma 4.2,

$$
L\left|[a, k]=\phi_{1}\right|[a, k] \sim \phi_{2}|[c, k]=L|[c, k] .
$$

Hence by Lemma $4.1, a=c$ and $\psi$ is trivial. Thus both $\phi$ and $\psi$ are equivalent to powers of $\phi$.

Now assume the proposition is true for all $n<n_{0}$. Suppose $\phi$ and $\psi$ are nontrivial and not equivalent. We may assume without loss of

3 Note that $\phi \psi \simeq \psi \phi$ so that by Theorem $3.1 \phi \psi \rightarrow \psi \phi$ and condition (3) above is satisfied. so that $N\left(F \mid s^{+}, \epsilon, A\right)=N\left(F \mid s^{-}, \epsilon, A\right)$. 


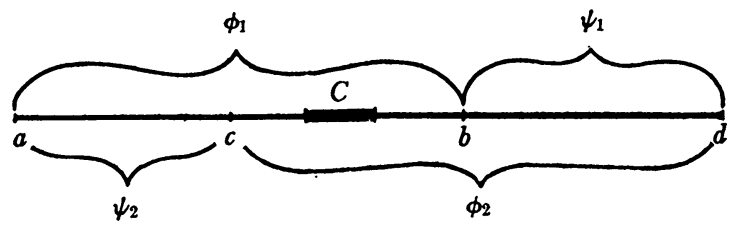

generality that $c<b$. Using arguments similar to those used in the case $n=1$, it can be shown that there must be $(\epsilon, p)$ sets for $L \mid \alpha$ in each of the intervals $[a, c],[c, b],[b, d]$. We define $\eta=L \mid[c, b]$. Then $\eta$ and $\psi$ commute, and since $\psi_{2} \eta=\phi_{1}$ is normal, we may apply the inductive hypothesis. The number of $(\epsilon, p)$ sets for $\phi_{1}$ is less than $n_{0}$, so that $\psi$ and $\eta$ are equivalent to powers of some loop $\mu$. It follows that $\phi$ and $\psi$ are equivalent to powers of the same loop $\mu$.

Now that we know that $\phi$ and $\psi$ are equivalent to powers of $\mu$, it follows that $f$ and $g$ are equivalent to powers of $h=\lambda \mu \lambda *$, and the theorem is proved.

Appendix. The definition of Fréchet equivalence used here is not quite the standard definition. In order to show that the relation is transitive it is necessary to prove the following lemma which we have been unable to find in the literature.

Lemma. If $f$ and $g$ are monotone order-preserving maps of the unit interval I onto itself, then there exist monotone order-preserving maps $\phi$ and $\psi$ of $I$ onto I such that $f \phi=g \psi$.

Proof. Let $F$ be the set of points $t \in I$ such that either $f^{-1}(t)$ or $g^{-1}(t)$ is a nondegenerate interval, and let $D$ be the union of $F$ with all of the rational points of $I$. We note that $D$ is countable, order-dense, and has two extreme elements.

Let $C$ be the Cantor middle-third set on $[1 / 3,2 / 3]$, and let $S$ be the complement of $C$ in $I$. Let $E$ be the set whose members are closures of components of $S$. We order $E$ by $J_{1}<J_{2}$ if $t_{1} \in J_{1}$ and $t_{2} \in J_{2}$ imply $t_{1}<t_{2}$. Then $E$ is denumerable, order-dense, and has two extreme elements, so that there exists a $1-1$ order-preserving function $h: E \rightarrow D$.

If $J \in E$, we define $\phi$ to be a linear order-preserving map of $J$ onto $f^{-1} h(J)$, and $\psi$ to be such a map of $J$ onto $g^{-1} h(J)$. Now $f^{-1}(D)$, $g^{-1}(D)$, and the set $G$ which is the union of the elements of $E$ are all dense in $I$. $\phi$ maps $G$ onto $f^{-1}(D)$ and $\psi$ maps $G$ onto $g^{-1}(D)$. Hence $\phi$ and $\psi$ can be extended to maps of $I$ onto $I$, and the resulting maps satisfy the conditions of the lemma. 


\section{BiBLIOGRAPHY}

1. M. L. Curtis and M. K. Fort, Jr., Homotopy groups of one-dimensional spaces, Proc. Amer. Math. Soc. vol. 8 (1957) pp. 577-579.

2. S. Eilenberg and S. MacLane, Relations between homology and homotopy groups of spaces, Ann. of Math. vol. 46 (1945) pp. 480-509.

3. M. K. Fort, Jr., Mappings of $S^{1}$ into one-dimensional spaces, Illinois J. Math. vol. 1 (1957) pp. 505-508.

4. H. B. Griffiths, Infinite products of semigroups and local connectivity, Proc. London Math. Soc. (1956) pp. 455-480.

UNIVERSITY OF GEORGIA

\section{ON ESSENTIAL FIXED POINTS}

\section{J. M. MARR}

The purpose of this note is to furnish an affirmative answer to a question posed at the Summer Institute on Set Theoretic Topology held at the University of Wisconsin in 1955. Let $X^{\boldsymbol{x}}$ denote the space of continuous functions of $X$ in to $X$ topologized by the compact open topology. A fixed point $p$ of a map $f \in X^{\boldsymbol{x}}$ is called essential if for each neighborhood $U$ of $p$ there is a neighborhood $N$ of $f$ such that if $g \in N$, then $g$ has a fixed point in $U$.

THEOREM. If $X$ is a compact Hausdorff space which has the fixed point property, then there is an $f \in X^{\boldsymbol{x}}$ such that each fixed point of $f$ is essential.

Proof. Let $x_{0}$ be any element of $X$, and consider the map $f \in X^{x}$ where $f(X)=x_{0}$. Let $U$ be any neighborhood of $x_{0}$. Then $N$ $=\{g: g(X) \subset U\}$ is a neighborhood of $f$ with the property that each $g \in N$ has a fixed point in $U$. Therefore $x_{0}$ is an essential fixed point of $f$. Since $x_{0}$ is the only fixed point of $f, f$ is the required map.

Kansas State College

Received by the editors June 16, 1958. 